\title{
Fast Energy Transport in Droplet Evaporation
}

\author{
Long Li*, Wenyuan Xie, Guang Zhang \\ Qian Xuesen Laboratory of Space Technology \\ NO. 104 Youyi Road, Haidian District, Beijing 100094, China \\ lilong@qxslab.cn; xiewenyuan@qxslab.cn; zhangguang@qxslab.cn
}

\section{Extended Abstract}

Droplet evaporation is one of the most effective approaches for heat and mass transfer and has tremendous applications in printing and coating processes[1,2], purification and desalination[3,4], surface patterning[5-8] and DNA mapping[9]. The cooling effect of evaporation from both perspiration and exhaled moisture plays a key role in the physiological regulation of body temperature. Particularly, energy dissipation through evaporation cooling in heat pipes is responsible for over $85 \%$ of the total thermal load in spacecraft thermal management[10-12]. Here evaporation removes large amount of heat and subsequently reduces the local temperature due to its endothermic attribute. However, recent studies have found unexpected condensation rather than evaporation in the heated region of heat pipe under superheated conditions[13]. This deviates from the conventional knowledge and may cause severe reliability issues. While the understanding of contact line dynamics and substrate effect on evaporation have been studied in great detail, few work has been done to study the susceptibility of temperature field and the underlying mechanisms for energy transport during the evaporation process.

Heat and mass transport in evaporating droplet are fundamentally complex due to the phase change process: the transport patterns of energy and mass are tightly coupled with locally confined interactions such like thermocapillary convection, surface tension and interfacial diffusion. Conventional wisdom on the investigation of droplet evaporation mainly focuses on the wettability and cooling effect of the evaporating sessile droplet. Many strategies have been undertaken to understand the contact line dynamics of a sessile droplet and several evaporation modes have been reported: the constant contact line mode where contact angle decreases; the constant contact angle mode, in which the contact line keeps decreasing; and the combination of the two. Recently, by tuning the surface energy and surface roughness using micro-/nanofabrication techniques, textured surfaces are developed to study the micro behavior of droplet wettability. Micro wettability states such like Wenzel and Cassie states are confirmed to affect the evaporation. All of these studies show good agreement with the diffusion evaporation model which neglects the thermal effects. Practically, thermal effects of the substrate and droplet during evaporation are often more important. It is found that motion of contact line is mainly caused by disturbance from thermocapillary convections in the contact area, which subsequently drives the dissipation of energy. Theoretically, thermocapillary convection in an evaporating droplet originates from the locally heterogeneous temperature distribution. Therefore, energy transport to the droplet interface can be promoted under the evaporation cooling and convective mixing effects. Typically here energy transport ratio of thermocapillary to evaporation latent heat is about 0.4 to 0.6 for spontaneous natural evaporation. For evaporation under high heating fluxes, the temperature conditions change rapidly and are sensitive to small disturbances along the contact-line, which makes the transport phenomena even more complicated.

In this work, we show that a fast energy dissipation route can be formed in the high thermal flux aided droplet evaporation. Experiments show that temperature of the droplet is lower than the bare substrate for evaporations with lower substrate temperatures, $T_{s}<36.8{ }^{\circ} \mathrm{C}$. As the temperature of substrate further increases, temperature of the droplet will be $5-14 \%$ higher than that of the bare substrate. Numerical simulations are performed to understand the mechanisms and confirm the experimental findings. Transition of dominated energy transport by thermal conduction and latent heat dissipation of phase change to Marangoni convection was found to be the main incentive. The present study provides insights into the understanding of evaporation in high thermal flux conditions and offers opportunities for thermal management under extreme heat flux density. 


\section{References}

[1] H. Kim, F. Boulogne, E. Um, I. Jacobi, E. Button, and H. A. Stone, "Controlled uniform coating induced by multiple Marangoni flows and surface-adsorbable macromolecules," Phys. Rev. Lett., vol. 116, pp. 124501, 2016.

[2] M. F. Zhao and X. Yong, "Nanoparticle motion on the surface of drying droplets," Phys. Rev. Fluid, vol. 3, pp. 034201, 2018.

[3] L. Huang, J. X. Pei, H. F. Jiang, and X. J. Hu, "Water desalination under one sun using graphene-based material modified PTFE membrane Desalination," Desalination, vol. 442, no. 1, 2018.

[4] V. Jaiswal, A. R. Harikrishnan, G. Khurana, and P. Dhar, "Ionic solubility and solutal advection governed augmented evaporation kinetics of salt solution pendant droplets," Phys. Fluid, vol. 30, pp. 012113, 2018.

[5] R. D. Deegan, O. Bakajin, T. F. Dupont, G. Huber, S. R. Nagel, and T. A. Witten, "Capillary Flow as the Cause of Ring Stains From Dried Liquid Drops,” Nature, vol. 389, pp. 827, 1997.

[6] T. P. Ding, K. Liu, J. Li, G. B. Xue, Q. Chen, L. Huang, B. Hu, and J. Zhou, "All-Printed Porous Carbon Film for Electricity Generation from Evaporation-Driven Water Flow," Adv. Function. Mater., vol. 27, pp. 1700551, 2017.

[7] W. B. Li, D. Lan, and Y. R. Wang, "Dewetting-mediated pattern formation inside the coffee ring," Phys. Rev. E., vol. 95, pp. 042607, 2017.

[8] P. J. Yunker, T. Still, M. A. Lohr, and A. G. Yodh, "Suppression of the coffee-ring effect by shape-dependent capillary interactions," Nature, vol. 476, pp. 308, 2011.

[9] J. P. Jing., "Dynamic regulation of mitochondrial function by glucocorticoids," Proc. Natl. Acad. Sci. U.S.A., vol. 95, pp. 8046, 1998.

[10] K. V. Paiva, M. B. H. Mantelli, and L. K. Slongo, "Experimental testing of mini heat pipes under microgravity conditions aboard a suborbital rocket," Aerospace Sci. Technol., vol. 45, pp. 367, 2015.

[11] A. H. Hosseinloo, F. F. Yap, and E. T. Chua, "Random vibration protection of a double-chamber submerged jet impingement cooling system: A continuous model," Aerospace Sci. Technol., vol. 35, no. 29, 2014.

[12] H. U. Oh, S. Shin, and C. W. Baek, "Thermal control of spacebome image sensor using heat pipe cooling system," Aerospace Sci. Technol., vol. 29, pp. 394, 2013.

[13] A. Kundan, T. T. T. Nguyen, J. L. Plawsky, P. C. Wayner, D. F. Chao, and R. J. Sicker, "Condensation on Highly Superheated Surfaces: Unstable Thin Films in a Wickless Heat Pipe,” Phys. Rev. Lett., vol. 118, pp. 094501, 2017. 